\title{
Fusion of strings vs. percolation and the transition to the quark gluon plasma
}

\author{
M.A.Braun * C.Pajares \\ Departamento de Física de Partículas, \\ Universidade de Santiago de Compostela, \\ 15706-Santiago de Compostela, Spain \\ and J.Ranft \\ INFN Laboratorio Nazionale del Gran Sasso
}

July 1997

\begin{abstract}
.
In most of the models of hadronic collisions the number of exchanged colour strings grows with energy and atomic numbers of the projectile and target. At high string densities interaction between them becomes important, which should melt them into the quark-gluon plasma state in the end. It is shown that under certain reasonable assumptions about the the string interaction, a phase transition to the quark gluon plasma indeed takes place in the system of many colour strings. It may be of the first or second order, depending on the particular mechanism of the interaction. The critical string density is about unity in both cases. In the latter case the percolation of strings occurs above the critical density. The critical density may have been already reached in central $\mathrm{Pb}-\mathrm{Pb}$ collisions at $158 \mathrm{~A} \mathrm{GeV}$.
\end{abstract}

\section{US-FT/24-97}

* On leave of absence from the Department of High Energy Physics, University of St. Petersburg, 198904 St. Petersburg, Russia. 


\section{Introduction}

Multiparticle production at high energies is currently (and successfully) described in terms of colour strings stretched between the projectile and target [1-3]. Hadronizing these strings produce the observed hadrons. In the original version the strings live independently and the observed spectra are just the sum of individual string spectra. However, with growing energy and/or atomic number of colliding particles, the number of strings grows and one expects that interaction between them becomes essential. This problem acquires still more importance, considering the idea that at high enough energy collisions of heavy enough nuclei may produce the quark-gluon plasma (QGP). The interaction between strings then has to make the system evolve towards the QGP state.

In our earlier publications we proposed a simple probabilistic model of string fusion [4], which was later realized as a Monte-Carlo algorithm [5]. It turned out to be quite successful in explaining some peculiar features of multiparticle production, such as heavy flavour enhancement at high energies and production of particles outside the kinematical limits of nucleon-nucleon collisions [6,7]. It did not however seem to predict any phase transition in the colour string system, which could be interpreted as a transition to the QGP.

In a recent publication it was noted that the string density reached in $\mathrm{Pb}-\mathrm{Pb}$ collisions at present energies is of the same order of magnitude as the critical density for the percolation of overlapping discs [8]. This could mean that strings may evolve to the QGP via the percolation process. It however remained not clear how the percolation can be incorporated into a more general picture of string interaction (and fusion), and why simple models of this interaction (in particular, the one proposed in [4]) do not lead to a phase transition. Also one would like to know possible alternatives to the percolation to be able to study from the experimental data the particular way the hadronic matter chooses to go into the QGP state. This is also related to the possible signatures of the percolation or of any other phase transition.

This note intends to discuss these poroblems. Essentially our conclusions are as follows. We show that the model of Ref. [4] indeed lacks any phase transition. Its absence is due to a particular fusion mechanism chosen (Sec. 2). A slight change in this mechanism gives a very similar and also explicitly solvable model, in which there is a clear phase transition to the QGP. This transition is of the 1st order (Sec. 3). The percolation occupies an intermediate place between the two models in what concerns the interaction mechanism. Its predictions for observable quantities are discussed in Sec. 4. Sec. 5 contains some conclusions.

This note is centered on the analytical treatment of the problem of string interaction. Such questions as string formation and their hadronization, which require Monte Carlo simulations, remained outside its scope. As a consequence we shall study only very primitive observables, 
whose behaviour under string fusion can be predicted on plausible assumptions: the total (charged) multiplicity and the total number of strings. The latter shows itself in forwardbackward correlations [9]. We shall study the behaviour of the system of a fixed number $N$ of strings under their interactions. In practice, of course, the number $N$ fluctuates, its average value growing with energy. This will undoubtedly smooth all irregularities introduced by the phase transition and make its detection still more difficult.

\section{Fusion of strings in the model [4]}

In this section we briefly review the model proposed in [4] from a somewhat novel point of view, looking for a possible sign of a phase transition in it. It was assumed in the model that strings fuse as soon as their positions in the transverse space coincide. The probability of fusion $p$ was assumed to be the ratio of the string own transverse area $v$ to the total interaction transverse area $V$. Of course one should consider $v$ as an effective area, which may be smaller than the geometrical one for small fusion probability. We also assumed that colours of fusing strings (i.e. colours placed at their ends) sum into the total colour of the resulting string. For the realistic QCD this summation should be performed according to the composition laws of the $S U(3)$ colour group. This can only be done within a Monte Carlo code. In the analytical approach of [4], as well as in the present note, to see qualitative predictions of string interaction, we adopt a simplified, Abelian, approach, taking that two strings with positive integer colours $n_{1}$ and $n_{2}$ fuse into a string of colour $n_{1}+n_{2}$. Ordinary string possess a unit colour $n=1$. The basis of the model is laid by the probability $P\left(\nu_{n}\right)$ to find $\nu_{n}$ strings of colour $n$, chosen to be

$$
P\left(\nu_{n}\right)=c \frac{Q^{N} p^{N-M}}{\prod_{n=1}\left(\nu_{n} !(n !)^{\nu_{n}}\right)} \prod_{k=1}^{M-1}(1-k p)
$$

Here $N=\sum_{n} n \nu_{n}$ is the fixed total colour of the strings which is also the number of ordinary strings originally produced; $M=\sum_{n} \nu_{n}$ is the total number of strings ("clusters", in terms of the statistical approach). In the following we pretend to change this probability. So it is instructive to analyse its foundation.

Since we shall study the behaviour of the system at fixed $N$, factors depending only on $N$ do not play any role and are actually a part of the normalization constant. The denominator factors in (1) are of the standard quantum statistical origin: $\nu_{n}$ ! takes into account the identity of $\nu_{n}$ strings of colour $n$ and each $n$ ! takes into account the identity of $n$ ordinary strings which fuse into a string of colour $n$. The number $N-M$ is, in fact, the number of 
fusions, hence the factor $p^{N-M}$. We come finally to the factor

$$
w=\prod_{k=1}^{M-1}(1-k p)
$$

which is the one that contains information about a particular way strings fuse.

To obtain it we made an additional assumption that fusion does not change the proper volume $v$ of strings, that is, all strings have the same volume $v$ irrespective of their colour (and consequently the "string tension" of a string with colour $n$ is $n^{2}$ greater than of the ordinary string). As a result, fused strings have the same probability to fuse as ordinary ones. The factor $w$ can then be obtained from a simple recurrency relation, which follows if one studies the system with one string of arbitrary color $k$ added. For this system to exist, it is necessary that the new string should not fuse with the rest. This leads to a relation

$$
w\left(\nu_{n}+\delta_{n k}\right)=w\left(\nu_{n}\right)\left(V-v \sum_{n} \nu_{n}\right) / V
$$

The second factor, equal to $1-p \sum_{n} \nu_{n}$, gives precisely the probability not to fuse with the rest. We chose to write it in terms of volumes to compare with the treatment in the following section. The recurrency relation (3) has evidently the solution (2)

Given the probability (1), one can calculate all observables of interest. Our main observable will be the average number of strings of colour $n,\left\langle\nu_{n}\right\rangle$. One easily finds (see Appendix for some details)

$$
\left\langle\nu_{n}\right\rangle \equiv \sum_{\left\{\nu_{n}\right\}} \nu_{n} P\left(\nu_{n}\right)=C_{N}^{n} p^{n-1}(1-p)^{N-n}
$$

It is just a binomial distribution in $n$. With the help of (4) we obtain the average total number of strings $\langle M\rangle$ :

$$
\langle M\rangle=\frac{1}{p}\left(1-(1-p)^{N}\right)
$$

From this last equation we see that $\langle M\rangle$ monotonously falls from $\langle M\rangle=N$ at $p=0$ to $\langle M\rangle=1$ at $p=1$, which clearly shows the effect of the fusion process but exhibits no sign of irregularity, which could indicate a phase transition.

One would also like to know the behaviour of the average multiplicity $\langle\mu\rangle$ of particles produced by the hadronizing strings as a function of $p$. To this purpose one has to make certain assumptions about the multiplicity $\mu_{n}$ corresponding to a string of colour $n$. On physical grounds the multiplicity should be proportional to the surface of the string area. With all strings having the same dimension this does not lead to any $n$ dependence. One may also expect some growth of the multiplicity with $n$ from the growing string tension. In the limiting case when the string of colour $n$ emits particles exactly as $n$ ordinary strings we shall have

$$
\langle\mu\rangle=\nu_{1} \sum_{n} n \nu_{n}=N \mu_{1}
$$


that is, one will not see any change in the spectra resulting from fusion. This is not a surprise, since with $\mu_{n}=n \mu_{1}$ fusion does not influence the multiplicity. In the opposite case, when a high-colour string emits particles exactly like an ordinary one, $\mu_{n}=\mu_{1}$ and one has

$$
\langle\mu\rangle=\mu_{1} M
$$

and with the growth of $p\langle\mu\rangle$ falls from $N \mu_{1}$ to $\mu_{1}$.

More generally, one can study the distribution in the number $m$ of produced particles, assuming that this distribution is $\lambda_{n}(m)$ for a string of colour $n$, satisfying

$$
\sum_{m} \lambda_{n}(m)=1 ; \quad \sum_{m} m \lambda_{n}(m)=\mu_{n}
$$

The resulting distribution $\Lambda(m)$ is then given by the average

$$
\Lambda(m)=\frac{\sum_{n} \lambda_{n}(m)<\nu_{n}>}{\sum_{n}<\nu_{n}>}
$$

From (6) one can calculate moments of the distribution $\Lambda(m)$. In particular the average number of emitted particles per string is

$$
<m>=<\mu>/<M>
$$

and the dispersion squared is

$$
D^{2}=\sum_{m} m^{2} \Lambda(m)-<m>^{2}
$$

Presenting

$$
\sum_{m} m^{2} \lambda_{n}(m)=\mu_{n}^{2}+d_{n}^{2}
$$

where $d_{n}$ is evidently the dispersion of the distribution in $m$ for a string of colour $n$, we split $D^{2}$ into an internal part, generated by this intrinsic dispersion, and an external one, generated by fluctuations in the distribution of strings in colour. This latter is given by

$$
D_{e x}^{2}=\sum_{n} \mu_{n}^{2}<\nu_{n}>-<m>^{2}
$$

and is presumably sensitive to a possible phase transition. It is positive and vanishes both for $p=0$ and $p=1$.

To have a clearer vision of the model from the statistical point of view, it is instructive to consider the "thermodinamical limit", which in our case means $V \rightarrow \infty$ and $N \rightarrow \infty$ with the density of strings $\rho=N / V$ constant. Evidently then $p=v / V \rightarrow 0$ and the relevant parameter describing string interaction is

$$
\eta=\rho v=N p
$$


In this limit we find from (4) and (5)

$$
\left\langle\nu_{n}\right\rangle / N=\frac{1}{\eta \sqrt{2 \pi n}} \exp \left(n-\eta+n \ln \frac{\eta}{n}\right)
$$

and

$$
\langle M\rangle / N=\frac{1}{\eta}\left(1-e^{\eta}\right)
$$

We observe that the model has correct scaling properties with $N$ : both $\left\langle\nu_{n}\right\rangle / N$ and $\langle M\rangle / N$ result independent of $N$ in the limit. From (12) we conclude that $\left\langle\nu_{n}\right\rangle$ has a maximum at $n=\eta$, so that with the growth of $\eta$ the distribution steadily shifts towards higher $n$. However there is no phase transition: the system does not change qualitatively as $\eta \rightarrow \infty$.

This circumstance is clearly seen from Figs. 1 to 3 , which show $\langle M\rangle,\langle\mu\rangle$ (in units $\mu_{1}$ ) and $D_{e x}^{2}$ (multiplied by 10) as a function of the parameter $\eta$ in the system of 50 ordinary strings. In this figures prediction of the model studied in this section are illustrated by the upper curves (marked 1). The curves are quite smooth and show a very slow fall off of $<M>$ and $\langle\mu\rangle$ and an equally slow growth of $D_{e x}^{2}$ with the growth of $\eta$.

The reason of this is, of course, evident. It resides in the assumption that the dimension of strings does not grow with colour. Strings with higher colour then fuse with the same probability as ordinary ones, so that fusion is not accelerated with the accumulation of high colour strings. This picture does not correspond to what one might expect on intuitive grounds. Presently we shall see, that once we substitute this assumption by a more physical one, the behaviour of the system changes radically and acquires the expected features with a clear phase transition at high enough $p$.

\section{Fusion of strings with the conservation of their volume: 1st order phase transition}

An alternative model, which also preserves the important quality of being explicitly soluble, is obtained if we assume that as string fuse, they conserve their proper volume. Namely, two ordinary strings fuse into a string with the volume $2 v$. Then generally a string of colour $n$ will have volume $n v$, and consequently the probability to fuse much higher. To obtain the relevant factor $w$ we construct a recurrency relation similar to (3). However in this case the factor $w$ will depend not only on the occupation numbers $\nu_{n}$ but also on the volume $V$, since

the probability of fusion depends on the volume available and the latter now depends on the colour of the added string. The recurrency relation we obtain is

$$
w\left(V, \nu_{n}+\delta_{n k}\right)=c w\left(V-k v, \nu_{n}\right)\left(V-k v-v \sum_{n} \nu_{n}\right)
$$


We have taken into account that the rest strings live in a smaller volume $V-k v$ left after a string of colour $k$ has been added.

Recurrency relation (14) can also be solved quite easily. We rewrite it as

$$
w\left(V+k v, \nu_{n}+\delta_{n k}\right)=c w\left(V, \nu_{n}\right)\left(V-v \sum_{n} \nu_{n}\right)
$$

Note that the factor $V-v \sum_{n} \nu_{n}$ is invariant under a simulataneous change $V \rightarrow V+k v$, $\nu_{n} \rightarrow \nu_{n}+\delta_{n k}$. Therefore, making $q_{k}$ such substitutions we obtain

$$
w\left(V+k q_{k} v, \nu_{n}+q_{k} \delta_{n k}\right)=c^{q_{k}} w\left(V, \nu_{n}\right)\left(V-v \sum_{n} \nu_{n}\right)^{q_{k}}
$$

and more generally

$$
w\left(V+v \sum_{k} k q_{k}, \nu_{n}+q_{n}\right)=c^{\sum_{k} q_{k}} w\left(V, \nu_{n}\right)\left(V-v \sum_{n} \nu_{n}\right)^{\sum_{k} q_{k}}
$$

Putting finally $\nu_{n}=0, w(V, 0)=1$ and $c=1 / V$ we obtain

$$
w=(1-p N)^{M}
$$

Thus the distribution in $\nu_{n}$ which desribes our new model is

$$
P\left(\nu_{n}\right)=c \frac{Q^{N} p^{N-M}}{\prod_{n=1}\left(\nu_{n} !(n !)^{\nu_{n}}\right)}(1-p N)^{M}
$$

One observes at once that this distribution makes sense only for $p \leq 1 / N$, or, in terms of the parameter $\eta$, Eq. (11)

$$
\eta \leq 1
$$

What happens if $p$ becomes greater than $1 / N$ ? The answer is evident. The total volume of strings $N v$ then exceeds the interaction volume $V$ and the only way for the strings to exist is to form a single string which spans all the interaction area. In other words, with the probability one, all string fuse into what may be viewed as the QGP plasma. This transition is of the first order, as we shall presently see considering the thermodynamic limit.

With the distribution (19) one can also calculate average occupation numbers in the (nearly) explicit form. One finds (see Appendix)

$$
\left\langle\nu_{n}\right\rangle=N \alpha C_{N}^{n} \frac{f_{N-n}(N \alpha)}{f_{N}(N \alpha)}
$$

and

$$
\langle M\rangle=\frac{f_{N+1}(N \alpha)}{f_{N}(N \alpha)}-N \alpha
$$

where

$$
\alpha=1 / \eta-1
$$


and $f_{N}(\alpha)$ is defined to be

$$
f_{N}(\alpha)=\left[\left(\frac{d}{d x}\right)^{N} e^{\alpha\left(e^{x}-1\right)}\right]_{x=0}
$$

It is a polinomial of order $N$.

These exact expressions are not very transparent. However one clearly sees from (22) that with the growth of $\eta$ from 0 to 1 (that is $p$ growing from 0 to $1 / N$ ) the average value $\langle M\rangle$ diminishes from $N$ down to exactly 1 . Indeed, at $\eta \rightarrow 0$ we have $\alpha \rightarrow \infty$ and only two highest powers of the polinomials $f_{N}$ have to be taken into account. One finds

$$
f_{N}(\alpha) \simeq \alpha^{N}+(1 / 2) N(N-1) \alpha^{N-1}+\ldots
$$

Putting this into (22) one obtains $\langle M\rangle=N$. At $\eta \rightarrow 1$ we have $\alpha \rightarrow 0$ and only the lowest term of $f_{n}$ contributes, which is $\alpha$ independently of $N$. From (22) one then finds $\langle M\rangle=1$.

To clearly see the phase transition we again take the thermodynamic limit $V, N \rightarrow \infty$ with the density $\rho$ and parameter $\eta$ fixed. Applying the saddle point technique (see Appendix) we then find a simple formula for $\left\langle\nu_{n}\right\rangle$

$$
\left\langle\nu_{n}\right\rangle / N=\alpha u^{n} / n !
$$

where $\alpha$ is given by (23) and $u$ is a solution of the transcendental equation

$$
\alpha u=e^{-u}
$$

Eq.(25) is nothing but a Poisson distribution (correctly normalized because of (26)). For the average $\langle M\rangle$ we obtain

$$
\langle M\rangle / N=1 / u-\alpha
$$

As we see, the system, again, possesses normal scaling properties with respect to the volume: the right-hand sides of (25) and (27) are independent of $N$.

From (25) one concludes that there is no infinite cluster for $\eta$ smaller than the critical value $\eta_{c}=1$, since $\nu_{\infty}=0$. At $\eta=\eta_{c}, \alpha$ goes to zero and $u$ becomes infinite, so that Eq.(25) ceases to exist. At $\eta>\eta_{c}$ we have a single infinite cluster and no finite ones. Thus if $P_{f(\infty)}$ are the probabilities to find a finite (infinite) cluster we have

$$
\begin{gathered}
P_{f}=1, \quad P_{\infty}=0, \quad 0 \leq \eta \leq \eta_{c}=1 \\
P_{f}=0, \quad P_{\infty}=1, \quad \eta \geq \eta_{c}=1
\end{gathered}
$$

This situation corresponds to a 1 st order phase transition at $\eta=\eta_{c}=1$. Slightly below the phase transition point we have

$$
\langle M\rangle / N=\frac{1}{\left|\ln \left(\alpha \ln \frac{1}{\alpha}\right)\right|}, \quad \alpha \simeq 1-\eta \rightarrow 0
$$


so that the behaviour near the critical point is logarithmic, and not power-like, as for the 2nd order phase transitions.

As to the multiplicity, it is reasonable to assume that it is proportional to the emitting surface and therefore to $\sqrt{n}$ for the string of colour $n$. Then the observed average multiplicity will be

$$
\langle\mu\rangle=\mu_{1} \sum_{n} \sqrt{n}\left\langle\nu_{n}\right\rangle
$$

$\mu_{1}$ being as before, the multiplicity for a single ordinary string. The sum (29) cannot be done analytically for finite $N$. For large $N$ the saddle point method gives

$$
\langle\mu\rangle / N=\mu_{1} / \sqrt{u}
$$

where $u$ is the solution of (26). This expression is valid for not very small $u$ : evidently for $\eta=0$ and consequently $u=0$ we have

$$
\langle\mu\rangle / N=\mu_{1}
$$

At $\eta$ close to the critical point we have

$$
\langle\mu\rangle / N=\frac{\mu_{1}}{\sqrt{\left|\ln \left(\alpha \ln \frac{1}{\alpha}\right)\right|}}
$$

logarithmically small, as the number of strings. Above the phase transition the whole interaction area acts as a single emitter and we have

$$
\langle\mu\rangle / N=\frac{\mu_{1}}{\sqrt{N}}
$$

so that the multiplicity is down by a (large) factor $\sqrt{N}$.

These features of $\langle M\rangle$ and $\langle\mu\rangle$ are clearly seen in Figs. 1 and 2 (curves marked 2). One finds a sharp drop of both $\langle M\rangle$ and $\langle\mu\rangle$ at the phase transition point $\eta=1$. Substantially below the transition point the fall off of both these quantities is quite slow and for $\eta$ below 0.5 predictions of the model practically coincide with those of the preceding section. The same conclusions can be drawn from Fig. 3 where the curve marked 2 shows the dispersion squared $D_{e x}^{2}$. Its sharp rise as $\eta$ approaches unity and subsequent fall to zero at $\eta$ exactly equal to unity are quite indicative of the first order phase transition.

\section{Percolation}

An alternative way to the formation of the QGP in the colour string system is the percolation. This is a purely classical mechanism. Consider projections of ordinary strings onto the transverse space as filled circles (discs) of area $v$. Let them be distributed uniformly in the 
interaction area $V$. Some of the discs may overlap and in this way form clusters of string matter. At some critical disc density $\rho$ a cluster appears which spans all the volume (an infinite cluster if the number of discs $N \rightarrow \infty$ ). This infinite string cluster may be identified with the GQP filling part of the total volume.

Comparing this mechanism with the string fusion we note that it occupies an intermediate position between the two scenarios discussed in Secs. 2 and 3, as far as the interaction mechanism is concerned. Indeed, in the percolation, strings are allowed to partially overlap, so that the volume of the resulting clusters varies between the two extremes: the volume of a single string, in the complete overlap case, and the sum of the interacting strings volumes, in the minimal overlap case. The two scenarios discussed above correspond just to these two extreme cases. An additional advantage of the percolation treatment is that it introduces various clusters for a given high colour $n>1$, thus taking into account (in a classical, not quantum way) possible excited states of fused strings, which should become numerous for large $n$. Unfortunately, the percolation cannot be treated analytically and nearly all the results known in the literature have been obtained by Monte Carlo simulations in the vicinity of the phase transition point.

The percolation phase transition is known to be of the 2 nd order. The critical value of the parameter $\eta$ introduced above is found to be [10]

$$
\eta_{c} \simeq 1.12
$$

This value is remarkably close to the critical value $\eta_{c}=1$ for the 1 st order phase transition in our model of string fusion discussed in Sec. 3. It is also known that the fraction $\phi$ of the volume, occupied by strings is determined by the formula [11]

$$
\phi=1-e^{-\eta}
$$

which coincides with the fraction of the volume $\phi=v\langle M\rangle$ in our string fusion model of Sec. 2 (Eq. (13)). In the model of Sec. 3, of course, $\phi=1$, since the volume is conserved in this model and, in terms of percolating discs, no overlapping takes place.

Below the phase transition point, for $\eta<\eta_{c}$, there is no infinite cluster, so that

$$
P_{f}=(1 / N) \sum_{n} n \nu_{n}=1, \quad P_{\infty}=0
$$

Above the transition point, at $\eta>\eta_{c}$ an infinite cluster appears with a probability

$$
P_{\infty}=\theta\left(\eta-\eta_{c}\right)\left(\eta-\eta_{c}\right)^{\beta}
$$

The critical exponent $\beta$ can be calculated from Monte Carlo simulations. However the universality of critical behaviour, that is its independence of the percolating substrate, allows to 
borrow its value from lattice percolation, where [10]

$$
\beta=5 / 36
$$

The main quantity of interest to us will, as before, be the average occupation numbers $\left\langle\nu_{n}\right\rangle$. Their behaviour at all values of $\eta$ and $n$ is not known. From scaling considerations in the vicinity of the phase transition it has been found [12]

$$
\left\langle\nu_{n}\right\rangle=n^{-\tau} F\left(n^{\sigma}\left(\eta-\eta_{c}\right)\right), \quad\left|\eta-\eta_{c}\right|<<1, n>>1
$$

where $\tau=187 / 91$ and $\sigma=36 / 91$ and the function $F(z)$ is finite at $z=0$ and falls off exponentially for $|z| \rightarrow \infty$. Expression (38) is of limited value, since near $\eta=\eta_{c}$ the bulk of the contribution is still supplied by low values of $n$, for which (38) is not valid. However from (38) one can find non-analytic parts of various observables at the transition point. In particular, we find the singular part of $\langle M\rangle$ in the form

$$
\Delta\langle M\rangle=c\left|\eta-\eta_{c}\right|^{8 / 3}
$$

This singularity is quite weak: not only $\langle M\rangle$ itself but also its two first derivatives in $\eta$ stay continuous at $\eta=\eta_{c}$ and only the third blows up as $\left|\eta-\eta_{c}\right|^{-1 / 3}$.

A little stronger singularity is found in the average multiplicity. Assuming it again to be proportional to linear dimensions of strings in the transverse plane, that is, to $\sqrt{n}$, we get from $(38)$

$$
\Delta\langle\mu\rangle=c\left|\eta-\eta_{c}\right|^{101 / 72}
$$

So already the second derivative of $\langle\mu\rangle$ blows up at the transition point.

More detailed predictions for the total $\langle M\rangle$ and $\langle\mu\rangle$, and not only for their singular parts, can only be obtained from Monte Carlo simulations. For a system of 50 strings they are presented in Figs. 1 to 3. by the curves marked 3. One observes that the behaviour of both $\langle M\rangle$ and $\langle\mu\rangle$ is quite smooth and does not seem to show any sign of irregularity at the phase transition point $\eta \simeq 1.12$. This is of no wonder, considering very weak singularities (39) and (40) introduced by the phase transition. Nevertheless, on the whole, the behaviour of both quantities is very different comparing to the previous models. Below the phase transition the string fusion is much stronger in the percolation case, so that averages of both $M$ and $\mu$ result much smaller than in the fusion models of Secs. 2 and 3. Above the phase transition point these averages continue to fall off quite smoothly, so that they become substantially greater than for the 1st order transition (Sec. 3) but stay much smaller than in the model of Sec. 2 without a phase transition. This opens up some possibilities to study the interaction mechanism from the observed behaviour of $\langle M\rangle$ and $\langle\mu\rangle$. The dispersion curve in Fig. 3 is more informative. It shows a clear maximum at values of $\eta$ in the interval 1.1-1.5. The 
absolute value of the dispersion at the maximum is nearly two orders of magnitude higher than those for the fusion models of Secs. 2 and 3 (which are shown in Fig. 3 multiplied by 10). Such a high dispersion is typical for a phase transition. From Fig. 3 one can approximately determine the critical value $\eta_{c}$ to lie around 1.3. It is somewehat higher than the value 1.12 known in the literature, but the latter corresponds to infinitely many strings, and our results refer to only 50 .

\section{Conclusions}

We have shown that string fusion may lead to a phase transition into the QGP for high enough string density. We presented a particular completely solvable string fusion model in which this phase transition is of the 1st order. Percolation is another alternative, which has certain advantages in treating the details of string interaction. It is important that in both models the transition to QGP starts practically at the same value of the string density (corresponding to $\eta=1$ in the string fusion model and $\eta=1.12$ in the percolation model). So, irrespective of the interaction mechanisms, one can expect QGP formation at such densities. As pointed out in [8], this critical density seems to have been nearly reached in $\mathrm{Pb}-\mathrm{Pb}$ collisions already at present energies at SPS and should be considerably surpassed in future RHIC and LHC experiments.

We have dealt only with qualitative features of string interactions and transition to QGP, which allow for an analytical treatment. Detailed predictions require a Monte Carlo algorithm.

The two models of phase transition presented above seem to be complementary for such a program. The string fusion model of Sec. 3 is well suited for the hadronization part, since one can comparatively easily extend the standard formalism of string decay to strings of higher colour. In fact, this already has been included in the Monte Carlo string fusion scheme of Ref [5]. However the model is difficult to realize on the level of string production: somehow the original strings should be melted into the fused one of the same geometrical form, which is not at all easy in the essentially classical Monte Carlo approach. The percolation treatment, on the contrary, is quite easily and naturally realizable at the string formation stage, but meets with serious problems at the hadronization level, when one has to introduce the decay of complicated geometrical structures corresponding to percolating clusters. These problems are left for future studies. In any case, experimental signatures like $J / \Psi$ suppression in $\mathrm{Pb}-\mathrm{Pb}$ collisions, seem to indicate that percolation of strings already occurs [8]. 


\section{Acknowledgements}

This work has been done under the contract AE96-1673 from CICYT (Spain). M.B. and J.R.thank the University of Santiago de Compostela for hospitality and financial support.

\section{$7 \quad$ References}

1. A. Capella, U. P. Sukhatme, C.-I. Tan and J. Tran Thanh Van, Phys. Lett. B81 (1979) 68; Phys. Rep. 236 (1994) 225.

2.A.B.Kaidalov and K.A. Ter-Martirosyan, Phys. Lett. B117 (1982) 247.

3. B. Andersson, G. Gustafson and B. Nilsson-Almqvist, Nucl. Phys. B281 (1987) 289.

4. M.A.Braun and C.Pajares, Nucl. Phys. B390 (1993) 542, 549.

5. N. S. Amelin, M. A. Braun and C. Pajares, Phys. Lett. B306 (1993) 312; Z. Phys. C63 (1994) 507.

6. N.Armesto, M.A.Braun, E.G.Ferreiro and C.Pajares, Phys. Lett. 344B (1995) 301.

7. N.Armesto, M.A.Braun, E.G.Ferreiro, C.Pajares and Yu..M.Shabelski, Phys. Lett. 389B (1996) 78; Astropartcle Phys. 6 (1997) 329.

8. N.Armesto, M.A.Braun, E.G.Ferreiro and C.Pajares, Phys. Rev.Lett. 77 (1996) 3736.

9. N.Amelin, N.Armesto, M.A.Braun, E.G.Ferreiro and C.Pajares, Phys. Rev. Lett. 73 (1994) 2813.

10. M.B.Isichenko, Rev. Mod. Phys.64 (1992) 961.

11. V.K.S.Shante and S.Kirkpatrick, Adv. Phys. 20 (1971) 325.

12. D.Stauffer, Phys. Rep. 54 (1979) 2. 


\section{Appendix: Averages over the distributions (1) and (14)}

We illustrate our technique by calculating the normalization constant $c$ in the distibutions (1) and (19). We use the identity

$$
\delta_{k l}=\int \frac{d z}{2 \pi i z} z^{k-l}
$$

where the integration is supposed to go along a closed contour around the origin. Then for both distributions we obtain

$$
c^{-1}=(Q p)^{N} \sum_{M} \int \frac{d u}{2 \pi i u} u^{-N} \int \frac{d z}{2 \pi i z} z^{-M} p^{-M} w(N, M) \sum_{\left\{\nu_{n}\right\}} \frac{u^{\sum_{n} n \nu_{n}} z^{\sum_{n} \nu_{n}}}{\prod_{n=1}\left(\nu_{n} !(n !)^{\nu_{n}}\right)}
$$

where the sums over $\nu_{n}$ are now unconstrained and can be easily done. In fact, we have

$$
T_{n} \equiv \sum_{\nu_{n}} \frac{u^{n \nu_{n}} z^{\nu_{n}}}{\nu_{n} !(n 1)^{\nu_{n}}}=\exp \left(z u^{n} / n !\right)
$$

and the product over $n$ gives

$$
T=\prod_{n=1} T_{n}=\exp \left(z\left(e^{u}-1\right)\right)
$$

Integration over $z$ is trivially performed and gives

$$
\left(e^{u}-1\right)^{M} / M !
$$

Now we have to sum over all $M$. This summation is different for the two distributions (1) and (19). For (1) the factor $w$ is given by (2) and can be presented in the form

$$
w(M)=p^{M} M ! C_{1 / p}^{M}
$$

Then the sum over $M$ gives

$$
e^{u / p}
$$

For (19) the factor $w$ is given by (18). The summation over $M$ then gives a more complicated factor

$$
e^{N \alpha\left(e^{u}-1\right)}
$$

where $\alpha$ is given by (23).

Finally the integration over $u$ has to be done, which reduces to taking the $N$ th term of the expansion of expressions (45) or (46) around $u=0$. For the distribution (1) it is trivial and gives

$$
c^{-1}=Q^{N} / N !
$$


whereas for the distribution (19) one obtains

$$
c^{-1}=(Q p)^{N} f_{N}(N \alpha) / N !
$$

where $f_{N}$ is defined by (24).

Note that the polinomials $f_{N}(\alpha)$ can be easily calculated by a recurrency relation which relates the coefficients for $f_{N}$ and $f_{N+1}$. Indeed one notices that the derivatives of the function (46) have the structure

$$
(d / d u)^{N} e^{\alpha\left(e^{u}-1\right)}=e^{\alpha\left(e^{u}-1\right)} f_{N}\left(\alpha e^{u}\right)
$$

Differentiating once more, one gets a relation

$$
c_{N+1}^{(k)}=c_{N}^{(k-1)}+k c_{N}^{(k)}, 1 \leq k \leq N
$$

for the coefficients $C_{N}^{(k)}$ of the polinomial $f_{N}$. Also one evidently has $c_{N}^{(0)}=0$ and $c_{N}^{(N)}=1$. Thus, starting from $N=1$, one can successively calculate all polinomials $f_{N}$ using the recurrency relation (50).

Averages like $\left\langle\nu_{n}\right\rangle$ or $\langle M\rangle$ have the same representation as (42) with some simple additional factors and are calculated in a similar manner.

To study the limit $N \rightarrow \infty$ the integral representation for $f_{N}(N \alpha)$ is more convenient:

$$
f_{N}(\alpha)=\int \frac{d u}{2 \pi i u} u^{-N} e^{N \alpha\left(e^{u}-1\right)}
$$

Presenting the integrand in the form $\exp N E(u)$ we have at large $N$

$$
E \simeq \alpha\left(e^{u}-1\right)-\ln u
$$

so that the saddle point is determined by Eq. (26). Knowing the saddle point it is trivial to calculate the averages $\left\langle\nu_{n}\right\rangle$, since for them an extra factor $N \alpha u^{n} / n$ ! has to be introduced under the integral. Taking this factor at the stationary point one obtains Eq. (25). 


\section{$9 \quad$ Figure captions}

Fig.1. The average number of clusters $M$ in the system of 50 strings as a function of $\eta$. Curves 1, 2 and 3 correspond to models of Secs. 2, 3 and 4 respectively. The latter curve was obtained by Monte Carlo simulations.

Fig.2. The average multiplicity $\mu$ in units $\mu_{1}$ in the system of 50 strings as a function of $\eta$. Curves 1, 2 and 3 correspond to models of Secs. 2, 3 and 4 respectively. In all cases $\mu_{n}$ was assumed proportional to $\sqrt{n}$. The percolation curve (3) was obtained by Monte Carlo simulations.

Fig.3. The dispersion squared $D_{e x}^{2}$ in the system of 50 strings as a function of $\eta$. Curves 1 , 2 and 3 correspond to models of Secs. 2, 3 and 4 respectively. Curves 1 and 2 show values of $D_{e x}^{2}$ multiplied by 10 . Curve (3) was obtained from Monte Carlo simulations for $\langle M\rangle$ and $\langle\mu\rangle$ (Figs. 1 and 2). 


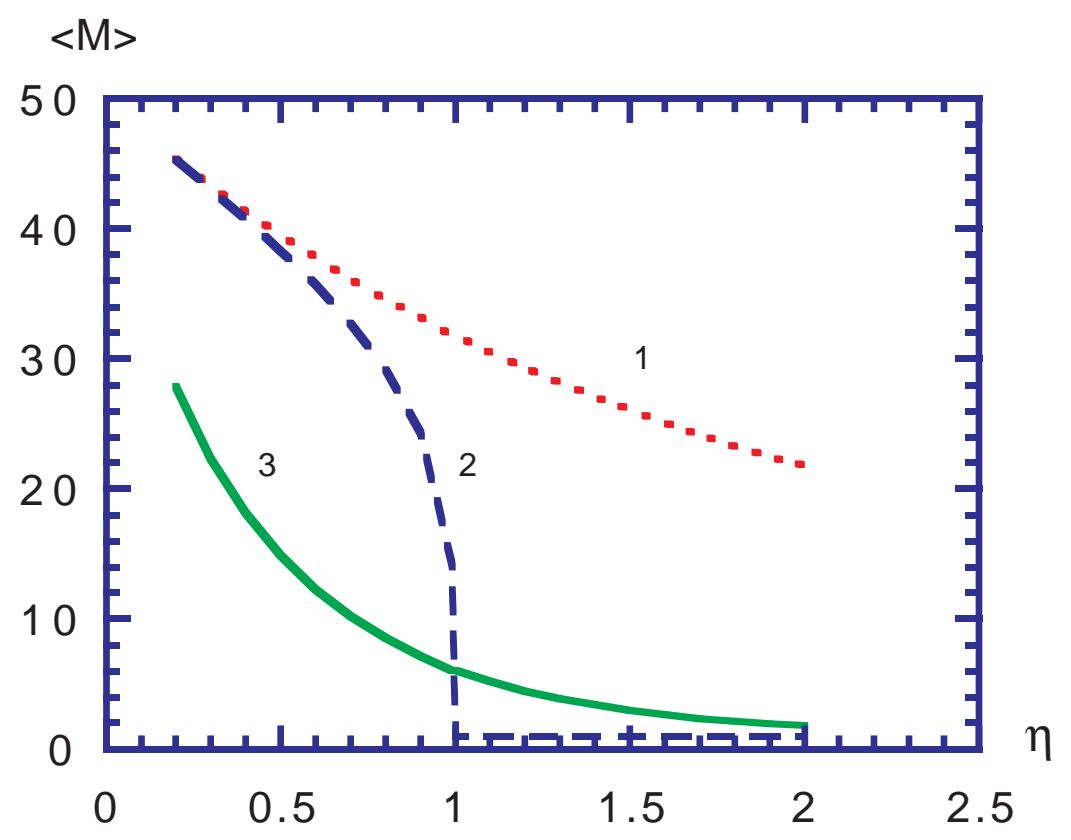

Fig. 1 




Fig. 2 


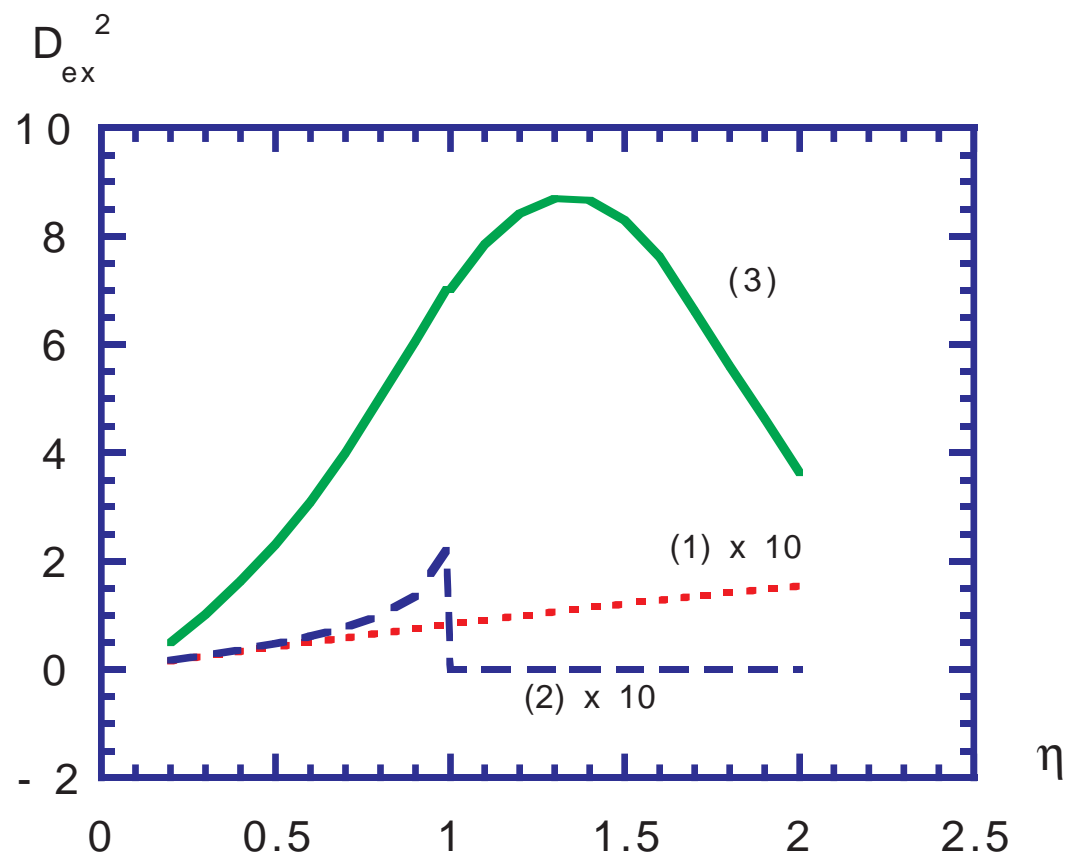

Fig. 3 